\title{
BOUNDS OF INFORMATION EXPENSES IN CONSTRUCTING PROJECTION METHODS FOR SOLVING ILL-POSED PROBLEMS
}

\author{
SERGEI G. SOLODKY AND EVGENIYA V.LEBEDEVA \\ Institute of Mathematics, National Academy of Sciences \\ 3 Tereschenkivska St., 01601 Kyiv, Ukraine
}

\begin{abstract}
An approach to constructing regularized projection methods to solve illposed problems is proposed. This approach is based on a modification of the Galerkin discretization scheme. It has been established that such a modification leads to a significant reduction of information expenses compared to other known methods.
\end{abstract}

2000 Mathematics Subject Classification: 65J20; 47A52.

Keywords: Ill-posed problems, regularization method, discretization, projection scheme, discrepancy principle.

\section{Introduction}

In a Hilbert space $X$ with inner product $(\cdot, \cdot)$ and norm $\|x\|=\sqrt{(x, x)}$ we shall consider the operator equation of the first kind

$$
A x=f .
$$

Suppose that in (1) $f \in \operatorname{Range}(\mathrm{A})$, Range $(\mathrm{A}) \neq \overline{\operatorname{Range}(\mathrm{A})}$ and instead of the right-hand side $f$ we are given only an approximation $f_{\delta} \in X$ such that $\left\|f-f_{\delta}\right\| \leqslant \delta, \delta>0$.

Our aim is to recover the normal solution $x^{\dagger}$ of (1) (i.e., the solution of (1) with minimal norm in $X$ ) with the best possible accuracy under the assumption

$$
x^{\dagger} \in M_{\nu, \rho}(A)=\left\{x: x=|A|^{\nu} v,\|v\| \leqslant \rho\right\} .
$$

Here $\rho>0$ is the known parameter, the unknown parameter $\nu$ belongs to the interval [1, $\left.\nu_{1}\right]$, $1<\nu_{1}<\infty,|A|=\left(A^{*} A\right)^{1 / 2}$ and $A^{*}$ is the adjoint operator of $A$.

Now the class of operators $A$ will be defined, on which we pursue the investigation. Thus, we take an orthonormal basis $E=\left\{e_{k}\right\}_{k=1}^{\infty}$ in $X$ and define a $P_{m}$ as the orthoprojector onto the linear span of elements $e_{1}, e_{2}, \ldots, e_{m}$, i.e., $P_{m} f=\sum_{k=1}^{m}\left(f, e_{k}\right) e_{k}$. By $\mathcal{H}^{r}, r=1,2, \ldots$, denote the class of compact linear operators $A$ with $\|A\| \leqslant 1$ acting in $X$ and satisfying the conditions

$$
\left\|\left(I-P_{m}\right) A\right\| \leqslant \beta_{r} m^{-r}, \quad\left\|A\left(I-P_{m}\right)\right\| \leqslant \beta_{r} m^{-r}
$$

at any $m=1,2, \ldots$ and some constant $\beta_{r}>0$.

An important example of (1) with $A \in \mathcal{H}^{r}$ is given by the first kind Fredholm integral equation

$$
A x(t) \equiv \int_{0}^{1} k(t, \tau) x(\tau) d \tau=f(t),
$$


where the integral operators $A, A^{*}$ act from $X=L_{2}(0,1)$ into the Sobolev space $W_{2}^{r}[0,1]$ of $r$-time differentiable functions. In particular, condition (3) is satisfied by the operators with kernels $k(t, \tau)$ that have $r$ bounded in $L_{2}(0,1)$ derivatives with respect to both variables. The following function systems can be used as the basis $E$ satisfying (3): the orthonormal system of Haars functions (at $r=1$ ), the subspace of trigonometric functions (in the case of 1-periodic coefficients $k(t, \tau), f(t))$ and the orthonormal system of Legendre polynomials considered on the interval $[0,1]$.

\section{Statement of the problem}

As is well known $[9, \mathrm{p} .14]$, the accuracy of recovery of solutions $x^{\dagger}$ filling the set $M_{\nu, \rho}(A)$ cannot be less than $\rho^{1 / \nu+1} \delta^{\nu / \nu+1}$. The class of equations (1) with the operators $A \in \mathcal{H}^{r}$ and the right-hand side $f \in A M_{\nu, \rho}(A)=\left\{f: f=A u, \quad u \in M_{\nu, \rho}(A)\right\}$, where $\nu \in\left(0, \nu_{1}\right]$, is denoted by $\Psi_{\rho, \nu_{1}}^{r}$. The goal of the present paper is to work out an efficient approach to the finite-dimensional solution of (1) which is economic in the sense of information expenses (see (8)) and allows to guarantee the optimal order of accuracy $O\left(\delta^{\nu / \nu+1}\right)$ on the class $\Psi_{\rho, \nu_{1}}^{r}$.

In [2], the authors discussed the same problem under the Tikhonov regularization with the source condition $f \in A M_{\nu, \rho}(A), 0<\nu \leqslant 1$. In some sense, the present paper is an extension of [2] with more general result.

\section{Proposed approach to solve (1)}

By virtue of compactness of the operator $A$ problem (1) is ill-posed. For this reason, constructing stable approximations of $x^{\dagger}$ requires the application of some special regularization methods (see [8]). Following [1], by the regularization method we shall mean an operator $R_{\alpha}=R_{\alpha}(A): X \rightarrow X$ such that as an approximate solution of (1) one takes the element $x_{\alpha}=R_{\alpha}(A) f_{\delta}$, where $\alpha>0$ is called the regularization parameter and $R_{\alpha}(A)=g_{\alpha}\left(A^{*} A\right) A^{*}$. Here $g_{\alpha}(\lambda)$ is the Borel measurable function on the interval [0,1] and satisfies the following conditions:

$$
\begin{aligned}
& \sup _{0 \leqslant \lambda<\infty} \lambda^{\nu}\left|1-\lambda g_{\alpha}(\lambda)\right| \leqslant \chi_{\nu} \alpha^{\nu} \\
& \sup _{0 \leqslant \lambda<\infty} \lambda^{1 / 2}\left|g_{\alpha}(\lambda)\right| \leqslant \chi_{*} \alpha^{-1 / 2}
\end{aligned}
$$

where

$$
0 \leqslant \nu \leqslant \nu_{*}, \quad \nu_{1} \leqslant 2 \nu_{*}-1
$$

$\chi_{\nu}\left(\chi_{0}=1\right), \chi_{*}$ are some positive constants independent of $\alpha$, and $\nu_{*}$ is referred to as the qualification of the method $R_{\alpha}$. There are many well-known methods satisfying (4)-(6). In particular, we can mention Tikhonov's method (with $g_{\alpha}(\lambda)=(\alpha+\lambda)^{-1}$ at $\nu_{*}=1$ ), Landweber's method (with $g_{\alpha}(\lambda)=\lambda^{-1}\left[1-(1-\mu \lambda)^{1 / \alpha}\right]$ at $\nu_{*}=\infty, 0<\mu<2$ ), and Showalter's method (with $g_{\alpha}(\lambda)=\lambda^{-1}\left(1-\exp (-\lambda / \alpha)\right.$ ) at $\left.\nu_{*}=\infty\right)$.

To realize finite-dimensional approximations for equation (1), it is necessary first to discretize its coefficients. To this end, we propose a modified projection scheme of discretization, whose main point is to replace the operator $A$ and the element $f_{\delta}$ by their finite-dimensional analogues

$$
A_{n}=\sum_{k=1}^{n}\left(P_{2^{k}}-P_{2^{k-1}}\right) A P_{2^{2 n-k}}+P_{1} A P_{2^{2 n}}, \quad P_{2^{n}} f_{\delta}=\sum_{k=1}^{2^{n}}\left(f_{\delta}, e_{k}\right) e_{k}
$$


where the basis $E=\left\{e_{k}\right\}_{k=1}^{\infty}$ appears in the definition of $\mathcal{H}^{r}$. Hereinafter by the discrete information on (1) we mean a set of values of inner products

$$
\left(A e_{j}, e_{i}\right), \quad\left(f_{\delta}, e_{i}\right)
$$

that are needed to construct coefficients (7). To reduce the content of discrete information (8), we have to choose in the best way the discretization parameter $n$ depending on the known values of $\delta, r, \beta_{r}, \rho$ (see (10)).

Note that the modified projection scheme (7) was used first in [4] to solve equation (1) in the case of the known parameter $\nu=2$.

The proposed approach to the realization of finite-dimensional approximations to $x^{\dagger}$ consists in the following. An approximate solution $\hat{x}_{\alpha}$ is determined by the rule

$$
\hat{x}_{\alpha}=R_{\alpha}\left(A_{n}\right) P_{2^{n}} f_{\delta} .
$$

Here the regularization method $R_{\alpha}$ satisfies conditions (4)-(6), $A_{n}$ has the form of (7), and as the value of the discretization parameter, one takes the least integer $n>0$ such that

$$
2^{-2 r n} n \leqslant 2 \delta / c_{1},
$$

where $c_{1}=2^{2 r}\left(2^{2 r}-1\right)^{-1} \beta_{r}^{2} \rho$. As a stop rule will be used the discrepancy principle [3]. In other words the proposed computational procedure is stoped as soon as the parameter $\alpha$ satisfies the following condition

$$
b_{1} \delta \leqslant\left\|P_{2^{n}} f_{\delta}-A_{n} \hat{x}_{\alpha}\right\| \leqslant b_{2} \delta, \quad 2<b_{1} \leqslant b_{2} .
$$

The regularized projection method (7), (9)-(11) will be denoted as $\left(R_{\alpha}, A_{n}, b_{1}, b_{2}\right)$.

\section{Main result}

In what follows we shall need some properties of the approximation of the operator $A_{n}$.

Lemma 4.1. Let $A \in \mathcal{H}^{r}, x^{\dagger} \in M_{\nu, \rho}(A), \nu \in\left[1, \nu_{1}\right]$. If the discretization parameter $n$ is chosen according to (10), then

$$
\left\|A_{n} x^{\dagger}-P_{2^{n}} f_{\delta}\right\| \leqslant 2 \delta, \quad\left\||A|^{\nu}-\left|A_{n}\right|^{\nu}\right\| \leqslant z(\nu) c_{3} \delta^{\nu / \nu+1}
$$

where $c_{3}=2^{1-2 r}\left(1+2^{r+2}\right)\left(2^{2 r}-1\right)$ and the function $z(\nu)$ is bound in $(0, \infty]$.

Proof. Using Lemma 2 [7] and relation (10), we obtain

$$
\left\|A_{n} x^{\dagger}-P_{2^{n}} f_{\delta}\right\| \leqslant\left\|A_{n} x^{\dagger}-P_{2^{n}} f\right\|+\left\|P_{2^{n}}\left(f-f_{\delta}\right)\right\| \leqslant c_{1} 2^{-2 r n}+\delta \leqslant 2 \delta .
$$

With the help of Lemma 1 [7] and Lemma 4.1 [6] one can estimate the norm of the operator $|A|^{\nu}-\left|A_{n}\right|^{\nu}$. Namely, it holds

$$
\left\||A|^{\nu}-\left|A_{n}\right|^{\nu}\right\| \leqslant z(\nu)\left\|A^{*} A-A_{n}^{*} A_{n}\right\|^{\min \{\nu / 2,1\}} \leqslant z(\nu)\left(\left(\beta_{r}^{2}+c_{2} n\right) 2^{-2 r n}\right)^{\min \{\nu / 2,1\}}
$$

with $c_{2}=2^{r+2} \beta_{r}^{2}$. Keeping in mind (10), we have

$\left\||A|^{\nu}-\left|A_{n}\right|^{\nu}\right\| \leqslant z(\nu)\left(\frac{2\left(\beta_{r}^{2}+c_{2}\right)}{c_{1}} \delta\right)^{\min \{\nu / 2,1\}}= \begin{cases}z(\nu)\left(\frac{2\left(\beta_{r}^{2}+c_{2}\right)}{c_{1}} \delta\right)^{\nu / 2} \leqslant z(\nu) c_{3} \delta^{\nu / \nu+1} & \text { at } \nu \in[1,2), \\ z(\nu) \frac{2\left(\beta_{r}^{2}+c_{2}\right)}{c_{1}} \delta \leqslant z(\nu) c_{3} \delta^{\nu / \nu+1} & \text { at } \nu \in\left[2, \nu_{1}\right] .\end{cases}$

This completes the proof. 
Theorem 4.1. The optimal order of accuracy $O\left(\delta^{\nu / \nu+1}\right)$ is achieved within the framework of $\left(R_{\alpha}, A_{n}, b_{1}, b_{2}\right)$ on the class $\Psi_{\rho, \nu_{1}}^{r}$.

Proof. To establish the present statement, we apply the technique used earlier in proving Theorem 3.3 [6] and Theorem 4.1 [5]. We can write the representation for the error of method $\left(R_{\alpha}, A_{n}, b_{1}, b_{2}\right)$

$$
x^{\dagger}-\hat{x}_{\alpha}=R_{\alpha, n}\left(A_{n} x^{\dagger}-P_{2^{n}} f_{\delta}\right)+S_{\alpha, n} x^{\dagger},
$$

where $R_{\alpha, n}=g_{\alpha}\left(A_{n}^{*} A_{n}\right) A_{n}^{*}, S_{\alpha, n}=I-g_{\alpha}\left(A_{n}^{*} A_{n}\right) A_{n}^{*} A_{n}$. From (5) it follows

$$
\left\|R_{\alpha, n}\right\|=\left\|g_{\alpha}\left(A_{n}^{*} A_{n}\right) A_{n}\right\| \leqslant \chi_{*} \alpha^{-1 / 2} .
$$

Then

$$
\left\|x^{\dagger}-\hat{x}_{\alpha}\right\| \leqslant\left\|S_{\alpha, n} x^{\dagger}\right\|+2 \chi_{*} \alpha^{-1 / 2} \delta
$$

Using (4), we find

$$
\left\|I-A_{n} R_{\alpha, n}\right\|=\left\|I-g_{\alpha}\left(A_{n} A_{n}^{*}\right) A_{n} A_{n}^{*}\right\| \leqslant \sup _{0 \leqslant \lambda<\infty}\left|1-\lambda g_{\alpha}(\lambda)\right| \leqslant 1 .
$$

Hence with the help of (11) and the representation

$$
A_{n} S_{\alpha, n} x^{\dagger}=\left(A_{n}-A_{n} R_{\alpha, n} A_{n}\right) x^{\dagger}=\left(P_{2^{n}} f_{\delta}-A_{n} \hat{x}_{\alpha}\right)+\left(I-A_{n} R_{\alpha, n}\right)\left(A_{n} x^{\dagger}-P^{2^{n}} f_{\delta}\right),
$$

one obtains

$$
b_{1} \delta-\left\|A_{n} x^{\dagger}-P_{2^{n}} f_{\delta}\right\| \leqslant\left\|A_{n} S_{\alpha, n} x^{\dagger}\right\| \leqslant b_{2} \delta+\left\|A_{n} x^{\dagger}-P_{2^{n}} f_{\delta}\right\| .
$$

From the left-hand side of (14) and Lemma 4.1 it follows that

$$
b_{1} \delta-2 \delta \leqslant\left\|A_{n} S_{\alpha, n} x^{\dagger}\right\|, \quad \alpha^{-1 / 2} \delta \leqslant \alpha^{-1 / 2}\left(b_{1}-2\right)^{-1}\left\|A_{n} S_{\alpha, n} x^{\dagger}\right\| .
$$

To estimate the norm of the operator $A_{n} S_{\alpha, n}$, we apply the polar decomposition $A_{n}=$ $U\left(A_{n}^{*} A_{n}\right)^{1 / 2},\|U\|=1$. Then $A_{n} S_{\alpha, n}=U\left(1-\left|A_{n}\right| g_{\alpha}\left(\left|A_{n}\right|^{2}\right)\left|A_{n}\right|\right)\left|A_{n}\right|=U S_{\alpha, n}\left|A_{n}\right|$. Next, with the help of relation (4) and Lemma 4.1 we find

$$
\begin{gathered}
\alpha^{-1 / 2}\left\|A_{n} S_{\alpha, n} x^{\dagger}\right\|=\alpha^{-1 / 2}\left\|A_{n} S_{\alpha, n}|A|^{\nu} v\right\| \leqslant \\
\rho \alpha^{-1 / 2}\left\|A_{n} S_{\alpha, n}\left|A_{n}\right|^{\nu}\right\|+\left\|A_{n} S_{\alpha, n}\left(|A|^{\nu}-\left|A_{n}\right|^{\nu}\right)\right\| \leqslant \rho \alpha^{-1 / 2}\left\|U S_{\alpha, n}\left|A_{n}\right|^{\nu+1}\right\|+ \\
\left\|U S_{\alpha, n}\left|A_{n}\right|\right\|\left\|\left|\left\|\left.\right|^{\nu}-\left|A_{n}\right|^{\nu}\right\| \leqslant \rho \chi_{(\nu+1) / 2} \alpha^{\nu / 2}+\rho \chi_{1 / 2} c_{3} z(\nu) \delta^{\nu / \nu+1} .\right.\right.
\end{gathered}
$$

Let us estimate

$$
\left\|S_{\alpha, n} x^{\dagger}\right\|=\left\|S_{\alpha, n}|A|^{\nu} v\right\| \leqslant \rho\left\|S_{\alpha, n}\left|A_{n}\right|^{\nu}\right\|+\rho\left\|S_{\alpha, n}\right\|\left\||A|^{\nu}-\left|A_{n}\right|^{\nu}\right\| \leqslant \rho \chi_{\nu / 2} \alpha^{\nu / 2}+\rho z(\nu) c_{3} \delta^{\nu / \nu+1} .
$$

Substituting (16) into (15), we obtain

$$
\alpha^{-1 / 2} \delta \leqslant\left(b_{1}-2\right)^{-1}\left(\rho \chi(\nu+1) / 2 \alpha^{\nu / 2}+\rho \chi_{1 / 2} c_{3} z(\nu) \delta^{\nu / \nu+1}\right) .
$$

Using (12) and the above bounds for values $\alpha^{-1 / 2} \delta,\left\|S_{\alpha, n} x^{\dagger}\right\|$, we have

$$
\begin{gathered}
\left\|x^{\dagger}-\hat{x}_{\alpha}\right\| \leqslant \rho\left(\chi_{\nu / 2} \alpha^{\nu / 2}+z(\nu) c_{3} \delta^{\nu / \nu+1}+2\left(b_{1}-2\right)^{-1} \chi_{*}\left(\chi_{(\nu+1) / 2} \alpha^{\nu / 2}+\chi_{1 / 2} c_{3} z(\nu) \delta^{\nu / \nu+1}\right)\right)= \\
c_{4} \alpha^{\nu / 2}+c_{5} \delta^{\nu / \nu+1}
\end{gathered}
$$


where $c_{4}=\rho\left(\chi_{\nu / 2}+2 \chi_{*} \chi_{(\nu+1) / 2}\left(b_{1}-2\right)^{-1}\right), \quad c_{5}=\rho z(\nu) c_{3}\left(2 \chi_{*} \chi_{1 / 2}\left(b_{1}-2\right)^{-1}+1\right)$. If $\alpha \leqslant \delta^{2 / \nu+1}$ then the following relation holds:

$$
\left\|x^{\dagger}-\hat{x}_{\alpha}\right\| \leqslant\left(c_{4}+c_{5}\right) \delta^{\nu / \nu+1} .
$$

Thus, the statement of Theorem is proved for $\alpha \leqslant \delta^{2 / \nu+1}$.

For arbitrary $\alpha_{1}>0$ we take any function $g_{\alpha}$ satisfying conditions (4)-(6). It is known (see Lemma $3.2[6]$ ) that there is a constant $c_{*}>0$ such that for all $0 \leqslant \lambda \leqslant 1$ and $\alpha \geqslant \alpha_{1}$

$$
\left(1-\lambda g_{\alpha}(\lambda)\right)^{2} \leqslant c_{*}\left(1-\lambda g_{\alpha_{1}}(\lambda)\right)^{2}+\alpha_{1}^{-1}\left(\lambda\left(1-\lambda g_{\alpha}(\lambda)\right)\right)^{2} .
$$

Then

$$
\left\|S_{\alpha, n} x^{\dagger}\right\|^{2} \leqslant c_{*}\left(\left\|S_{\alpha_{1}, n} x^{\dagger}\right\|^{2}+\alpha_{1}^{-1}\left\|A_{n} S_{\alpha, n} x^{\dagger}\right\|^{2}\right) .
$$

Set $\alpha_{1}=\delta^{2 / \nu+1}$. Keeping in mind Lemma 4.1 and the right-hand side of (14), we find

$$
\alpha_{1}^{-1}\left\|A_{n} S_{\alpha, n} x^{\dagger}\right\|^{2} \leqslant\left(b_{2}+2\right)^{2} \delta^{2 \nu / \nu+1} .
$$

In view of (17), we obtain

$$
\left\|S_{\alpha_{1}, n} x^{\dagger}\right\|^{2} \leqslant\left(\rho \chi_{\nu / 2} \delta^{\nu / \nu+1}+\rho z(\nu) c_{3} \delta^{\nu / \nu+1}\right)^{2}=\delta^{2 \nu / \nu+1}\left(\rho \chi_{\nu / 2}+\rho z(\nu) c_{3}\right)^{2} .
$$

Substituting the above bounds for values $\alpha_{1}^{-1}\left\|A_{n} S_{\alpha, n} x^{\dagger}\right\|^{2}$ and $\left\|S_{\alpha_{1}, n} x^{\dagger}\right\|^{2}$ into (18), we get

$$
\begin{gathered}
\left\|S_{\alpha, n} x^{\dagger}\right\|^{2} \leqslant c_{*}\left(\left(\rho \chi_{\nu / 2}+\rho z(\nu) c_{3}\right)^{2} \delta^{2 \nu / \nu+1}+\left(b_{2}+2\right)^{2} \delta^{2 \nu / \nu+1}\right)= \\
c_{*}\left(\left(\rho \chi_{\nu / 2}+\rho z(\nu) c_{3}\right)^{2}+\left(b_{2}+2\right)^{2}\right) \delta^{2 \nu / \nu+1} .
\end{gathered}
$$

Since $\alpha>\alpha_{1}=\delta^{2 / \nu+1}$, it is not difficult to see that

$$
\alpha^{-1 / 2} \delta \leqslant \alpha_{1}^{-1 / 2} \delta=\delta^{\nu / \nu+1} .
$$

Let us substitute the bounds for values $\alpha^{-1 / 2} \delta$ and $\left\|S_{\alpha, n} x^{\dagger}\right\|$ into (12). Then

$$
\left\|x^{\dagger}-\hat{x}_{\alpha}\right\| \leqslant c_{6} \delta^{\nu / \nu+1}
$$

with $c_{6}=\left(c_{*}\left(\rho \chi_{\nu / 2}+\rho z(\nu) c_{3}\right)^{2}+\left(b_{2}+2\right)^{2}\right)^{1 / 2}+2 \chi_{*}$.

Thus, the relation

$$
\left\|x^{\dagger}-\hat{x}_{\alpha}\right\| \leqslant \delta^{\nu / \nu+1} \max \left\{c_{6}, c_{4}+c_{5}\right\}
$$

is valid at any $\alpha>0$. Theorem 1 is proved.

Corollary 4.1. To ensure the optimal order of accuracy $O\left(\delta^{\nu / \nu+1}\right)$ within the framework of $\left(R_{\alpha, n}, A_{n}, b_{1}, b_{2}\right)$ on the class $\Psi_{\rho, \nu_{1}}^{r}$, it is required to use $O\left(\delta^{-1 / r} \log ^{1+1 / r} \delta^{-1}\right)$ values of the inner products (8).

Proof. Introduce the quantity $\operatorname{Card}(n)$, which denotes the volume of inner products of the form $\left(A e_{j}, e_{i}\right)$ involved in constructing $A_{n}$. Obviously, the order of the total content of discrete information (8) required for the discretization scheme (7) is determined by Card $(n)$. Let us calculate

$$
\operatorname{Card}(n)=2^{2 n}(1+n / 2) .
$$

By virtue of the choice of the parameter $n$ (10) we have

$$
n 2^{-2 r n}=O(\delta), \quad 2^{2 n}=O\left(\delta^{-1 / r} n^{1 / r}\right) .
$$

Then

$$
\operatorname{Card}(n)=O\left(n n^{1 / r} \delta^{-1 / r}\right)=O\left(\delta^{-1 / r} \log ^{1+1 / r} \delta^{-1}\right)
$$




\section{Additional comments}

The traditional Galerkin method (i.e., $A_{m, l}=P_{m} A P_{l}$ ) was used in [6] to solve (1) with solutions belonging to the set $M_{\nu, \rho}(A)$. From the results of [6] it follows that the best choice of parameters $m$ and $l$ within the framework of the standard approach to the discretization gives the following bound:

$$
O\left(\delta^{-\left(3 \nu_{1}+1\right) /\left[2\left(\nu_{1}+1\right) r\right]}\right)
$$

of minimal information expenses of $(8)$ on the class $\Psi_{\rho, \nu_{1}}^{r}, 1<\nu_{1}<\infty$. A modification of the Galerkin scheme (different from (7) ) was used in [5] to solve equation (1) from $\Psi_{\rho, \nu}^{r}$, $\nu \in[1,2]$. By this modification one can reduce the information expenses of (8) up to

$$
O\left(\delta^{-13 / 12 r} \log \delta^{-1}\right)
$$

The last result was generalized on the class $\Psi_{\rho, \nu_{1}}^{r}, 1<\nu_{1}<\infty$, and improved on the logarithmic factor

$$
O\left(\delta^{-\left(5 \nu_{1}+3\right) /\left[4\left(\nu_{1}+1\right) r\right]}\right)
$$

in [7]. Comparison of the bound (19) with the above results proves the efficiency of the method $\left(R_{\alpha}, A_{n}, b_{1}, b_{2}\right)$.

\section{Numerical example}

The efficiency of the proposed approach to the solution of equation (1) is demonstrated with the example of the Fredholm integral equation

$$
\tilde{A} x(t) \equiv \int_{0}^{1} k(t, \tau) x(\tau) d \tau=f(t)
$$

where $x(t), f(t) \in L_{2}(0,1)$ and

$$
k(t, \tau)= \begin{cases}\tau^{2}(3 t-\tau) / 6, & 0 \leqslant \tau \leqslant t \leqslant 1 \\ t^{2}(3 \tau-t) / 6, & 0 \leqslant t \leqslant \tau \leqslant 1\end{cases}
$$

It is well known [10, Chapter 2, §15] that equation (20) arises in solving the following boundary value problem:

$$
f^{(I V)}=0, \quad f(0)=f^{\prime}(0)=f^{\prime \prime}(1)=f^{\prime \prime \prime}(1)=0,
$$

and the operator $\tilde{A}$ acts from $L_{2}(0,1)$ into $W_{2}^{4}[0,1]$. As the exact solution $x^{\dagger} \in M_{1,1}(\tilde{A})$, one takes the function

$$
x^{\dagger}(t):=|\tilde{A}| \cdot 1(t)=\frac{t^{4}}{8}+\frac{t^{2}\left(1-t^{2}\right)}{4}-\frac{t^{3}(1-t)}{6} .
$$

To realize the projection scheme (7), we use the orthonormal system of Legander's polynomials considered on the interval $[0,1]$. Equation (20) is solved with the help of Landweber's method

$$
x_{0}=0, \quad x_{l}=x_{l-1}-\mu \tilde{A}_{n}^{*}\left(\tilde{A}_{n} x_{l-1}-P_{2^{n}} f_{\delta}\right), \quad l=1,2, \ldots
$$


The calculations are performed at the error level $\delta=10^{-3}$. The discretization parameter $n$ is chosen in accordance with (10) and is equal to 3 .

The results of the test computation are presented in Table for various values of $b_{2}$ and $\mu$, where the symbol "it" is interpreted as the number of iteration steps and the symbol "error" denotes the accuracy of approximation in the $L_{2}(0,1)$ metrics. The graphs of the exact solution (bold line) and of the approximate solution obtained at $b_{2}=5 / 2$ and $\mu=3 / 2$ are plotted in Figure.

\begin{tabular}{|c|c|c|c|}
\hline$b_{2}$ & $\mu$ & error & it \\
\hline $5 / 2$ & 1 & 0.002261 & 238 \\
$5 / 2$ & $3 / 2$ & 0.00222 & 158 \\
$5 / 2$ & 2 & 0.00231 & 119 \\
$5 / 2$ & $5 / 2$ & 0.00231 & 95 \\
$5 / 2$ & 3 & 0.00229 & 79 \\
3 & 1 & 0.01471 & 136 \\
3 & $3 / 2$ & 0.01455 & 91 \\
3 & 2 & 0.01461 & 68 \\
3 & $5 / 2$ & 0.01478 & 54 \\
3 & 3 & 0.01473 & 45 \\
\hline
\end{tabular}

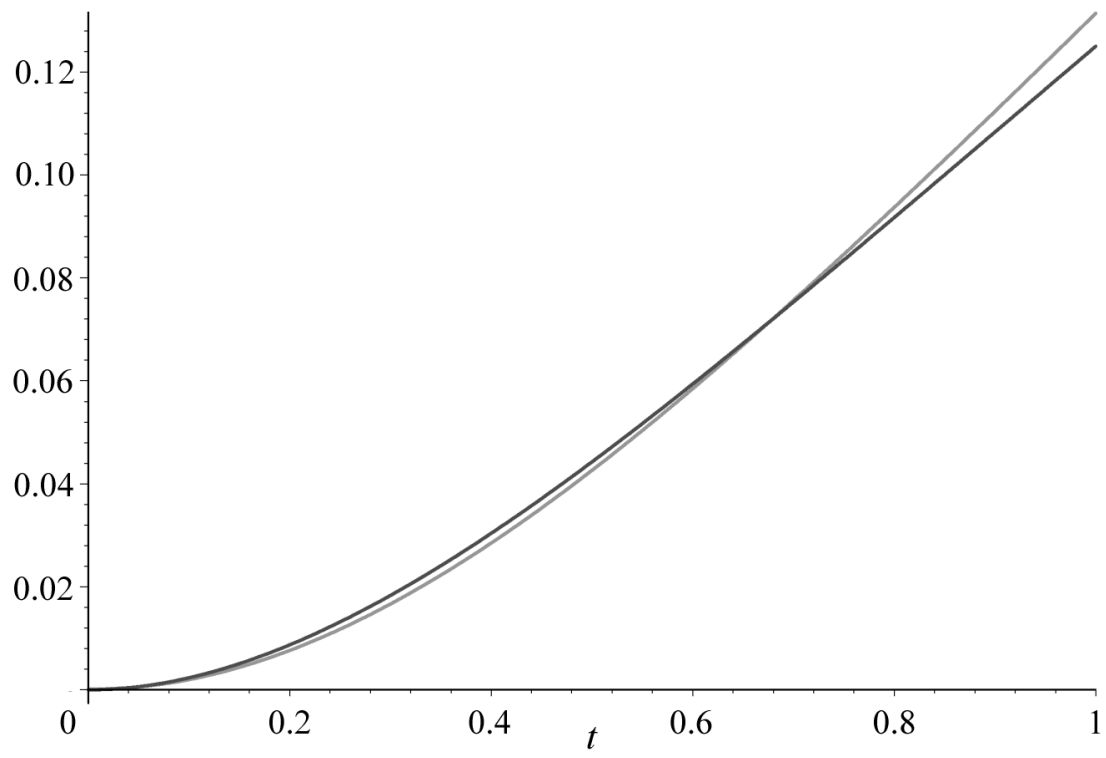

\section{Acknowledgment}

We wish to express our sincere gratitude to the anonymous referee for helpful comments and hints. This helped to make the presentation of this paper much clearer.

\section{References}

1. A. B. Bakushinski, A general method of constructing regularizing algorithms for a linear ill-posed equation in Hilbert Space, USSR Comput. Math. Math. Phys., 7 (1967), No. 3, pp. 672-677.

2. P. Maass, S. V.Pereversev, R. Ramlau, S. G. Solodky, An adaptive discretization for Tikhonov Phillips regularization with a posteriori parameter selection, Numer. Math., 87 (2001), pp. 485-502.

3. V. A. Morozov, On the solution of functional equations by the method of regularization, Sov. Math. Dokl., 7 (1966), pp. 414-417.

4. S. V.Pereverzev, Optimization of projection methods for solving ill-posed problems, Computing, $\mathbf{5 5}$ (1995), pp. 113-124.

5. S. V.Pereverzev and S. G. Solodky, An efficient discretization for solving ill-posed problems, Lect. Appl. Math., 32 (1996), pp. 643-649.

6. R. Plato and G. Vainikko, On the regularization of projection methods for solving ill-posed problems, Numer. Math., 57 (1990), pp. 63-79.

7. S. G. Solodky, A Generalized projection scheme for solving ill-posed problems, J. Inverse Ill Posed Probl., 7 (1999), No. 2. pp. 185-200.

8. A. N. Tikhonov and V. A. Arsenin, Solutions of ill-posed problems, Wiley, New York, 1977.

9. G. M. Vainikko and A. Yu. Veretennikov,Iteration procedures in ill-posed problems, Nauka, Moscow, 1986. [Russian]

10. M. L. Krasnov, A. I. Kiselev, G. V. Makarenko, Integral Equations. Second enlarged edition, Nauka, Moscow, 1976, in Russian. 